\title{
Génération de trajectoires de tréflage et d'usinage trochoïdal pour le vidage de poche
}

\author{
Matthieu Rauch ${ }^{\mathrm{a}}$ et Jean-Yves Hascoet \\ Institut de Recherche en Communications et Cybernétique de Nantes (IRCCyN) - UMR CNRS 6597, 1 rue de la Noë, BP 92101, \\ 44321 Nantes Cedex 03, France
}

Reçu le 15 mars 2007, accepté le 6 juillet 2007

\begin{abstract}
Résumé - Le vidage de poche est une problématique centrale du fraisage d'ébauche. Malgré les évolutions technologiques, aucune évolution majeure n'a été observée récemment concernant les trajectoires d'usinage de ces entités. Le tréflage et l'usinage trochoïdal sont de nouveaux types de stratégies qui ont trouvé des applications dans l'usinage d'ébauche des matériaux durs. Cet article vise à évaluer leur potentiel pour le vidage de poches dans des alliages d'aluminium. Des algorithmes de génération de trajectoires de tréflage et de trochoïdes ont ainsi été développés en cherchant un compromis entre productivité et qualité des surfaces obtenues. Une étude expérimentale a également été menée. Deux machines outils ont été utilisées, l'une sérielle à 3 axes, l'autre parallèle à 5 axes. Les résultats de cette étude conduisent à une meilleure connaissance de ces nouvelles stratégies d'usinage, en vue de leur application au fraisage d'ébauche d'alliages légers.
\end{abstract}

Mots clés : Vidage de poches / tréflage / usinage trochoïdal / définition de stratégies d'usinage grande vitesse

\begin{abstract}
Generation of plunging and trochoïdal toolpaths for pocket milling. Pocket milling is an essential issue in rough milling. Although machining capacities have been strongly modified, no significant evolution was observed about pocket milling trajectories. Trochoïds and plunge milling are new types of strategies which have found practical applications in hard materials rough milling. This paper aims at evaluating their potential in rough pocket milling of aluminium alloys. Algorithms were developed to generate trochoïdal and plunge cutting trajectories for pocket milling, with cycle time optimisation regarding to milled surfaces quality. An experimental campaign has also been done and focused both on productivity and quality issues. Two different high speed milling machine tools were tested: a serial threeaxis machine tool and a parallel kinematics five-axis machine tool. The results of this work leads to a better definition of the strengths and weaknesses of trochoïdal milling and plunge cutting strategies in aluminium alloys rough machining with the focus to optimize the choice of strategies.
\end{abstract}

Key words: Pocket milling / plunge cutting / trochoïdal milling / toolpath generation for high speed milling

\section{Introduction}

Le vidage de poches est une des problématiques centrales du fraisage d'ébauche. La principale contrainte associée est d'obtenir un débit de copeau maximal tout en produisant des surfaces de qualité suffisante pour faciliter le déroulement des opérations de finition. Pourtant, bien que de nombreux progrès aient été effectués en terme de dynamique et de puissance de machines-outils, avec notamment le développement de l'usinage grande vitesse, il

\footnotetext{
a Auteur pour correspondance :

matthieu.rauch@irccyn.ec-nantes.fr
}

n'y a pas eu d'amélioration notable en ce qui concerne la génération de trajectoires d'usinage dans les systèmes industriels. Ainsi, la grande majorité des logiciels de CFAO actuels ne propose comme stratégie de vidage de poche pratiquement que des trajectoires inspirées de zigzag ou de contour parallèle.

A contrario, le tréflage et les trajectoires trochoïdales sont des stratégies d'usinage innovantes qui ont trouvé des domaines d'application dans l'usinage des matériaux durs. En effet, le tréflage permet de réduire les vibrations et la flexion de l'outil car ce dernier travaille dans sa configuration la plus rigide; les efforts d'usinage peuvent 


\section{Nomenclature}

\begin{tabular}{|ll|}
\hline$D:$ & diamètre de l'outil, exprimé en mm \\
$d:$ & la profondeur de la poche, exprimée en mm \\
$\Delta_{\mathrm{p}}:$ & incrément de prise de passe radiale en tréflage, \\
& $\quad$ exprimé en mm \\
$\Delta_{\mathrm{t}}:$ & incrément de prise de passe radiale en usinage \\
& $\quad$ trochoïdal, exprimé en $\mathrm{mm}$ \\
$h:$ & la hauteur de remontée de l'outil, exprimée en mm \\
$L:$ & largeur de la trajectoire enveloppe de la trochoïde, \\
& $\quad$ exprimée en mm \\
$R:$ & distance entre A et B (Fig. 3), exprimée en mm \\
$t:$ & temps, exprimé en $\mathrm{s}$ \\
$V:$ & vitesse du point A (Fig. 3) exprimée en $\mathrm{m} . \mathrm{s}^{-1}$ \\
$\omega:$ & pulsation, exprimée en rad.s ${ }^{-1}$ \\
\hline
\end{tabular}

être mieux maîtrisés et la puissance d'usinage réduite en utilisant des stratégies trochoïdales. Par contre, ces deux types de trajectoires nécessitent une dynamique de machine élevée pour que leur productivité soit acceptable. C'est pourquoi elles n'ont pas été utilisées jusqu'à l'avènement des centres d'usinage à grande vitesse. De la même manière, les principaux logiciels de CFAO ne proposent des trajectoires de tréflage et d'usinage trochoïdal que depuis les toutes dernières années et leur implémentation reste très anecdotique.

Après un bref état de l'art sur le vidage de poches, l'article propose dans un premier temps d'identifier les principaux paramètres nécessaires à la définition de trajectoire de tréflage et d'usinage trochoïdal. Le potentiel de chaque stratégie est aussi donné, ainsi que le principe des algorithmes de génération développés. Ensuite, une comparaison de ces stratégies prometteuses avec les stratégies classiques est effectuée dans le cadre d'une étude expérimentale centrée à la fois sur des problématiques de productivité et de qualité des surfaces obtenues.

\section{Stratégies classiques}

Les deux types de trajectoires d'usinage les plus utilisés en fraisage d'ébauche sont le contour parallèle et le zigzag (Fig. 1). En zigzag, les trajectoires sont construites de manière élémentaire à partir de segments de droites parallèles entre eux et dont les extrémités sont obtenues à partir des contours de la poche. Pour le contour parallèle, on utilise les contours de la poche pour construire des trajectoires décalées les unes à l'intérieur des autres. Ces morceaux de trajectoires sont ensuite reliés entre eux par des liaisons. La poche est ainsi usinée à la manière d'une spirale, en suivant des courbes à équidistance des contours [1].

De nombreux travaux ont été publiés sur l'optimisation de ces deux types de stratégies d'usinage. Par exemple, les études sur le contour parallèle se sont focalisée sur les diagrammes de Voronoï [2], sur des approches
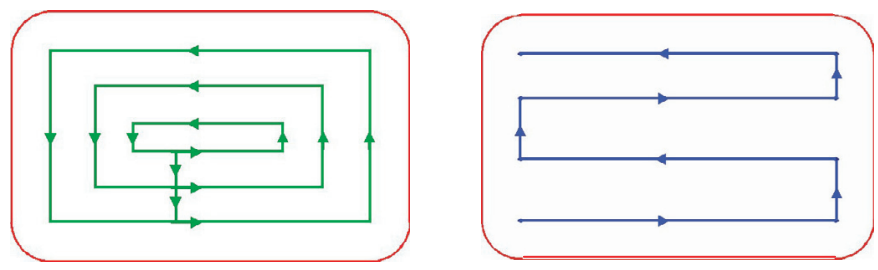

Fig. 1. Exemples de stratégies en contour parallèle et en zigzag.

par pixellisation [3] ou sur des décalages appairés [4]. Dans ses travaux, Pateloup propose une méthode intéressante basée sur les diagrammes de Voronoï et l'emploi de courbes B-splines lors des changements de direction, ceci dans le but de minimiser le temps d'usinage [5]. Pour ce qui est des stratégies en zigzag, l'accent a été mis sur l'optimisation de la direction de balayage, ceci afin de minimiser la longueur du trajet [6]. Plus récemment, les caractéristiques technologiques liées aux moyens de fabrications ont été intégrées dans la boucle d'optimisation. La géométrie des trajectoires a été modifiée pour limiter le nombre de discontinuités apparaissant à chaque changement de direction et qui peuvent réduire l'avance de manière conséquente [7]. Dans [8], Mawussi et al. proposent des éléments pour le choix de stratégie d'usinage pour le vidage de poche en s'appuyant sur des critères géométriques, les caractéristiques dynamiques du moyen utilisé doivent aussi être prises en compte. Après avoir testé plusieurs types de stratégies sur une machine outil, Kim et al. ont montré que les stratégies en zigzag sont plus productives que celles en contours parallèles [9].

Cependant, les stratégies développées sont toutes basées sur le zigzag ou le contour parallèle. Il est même surprenant qu'aucun autre type de stratégie innovante n'ait réussi à les surpasser bien que les moyens de production aient beaucoup évolués... avec notamment le développement des machines parallèles et des moteurs linéaires par exemple [10]. Certains auteurs voient néanmoins dans l'usinage trochoïdal et le tréflage des voies d'optimisation pour l'usinage d'ébauche $[11,12]$.

\section{Objectifs}

Lors de la préparation d'une opération d'usinage, l'expert en fabrication utilise son expérience et ses connaissances pour déterminer le bon mode opératoire, la meilleure stratégie FAO, l'outil le plus adapté, etc. Il n'y a donc pas de critère formel, tous ces choix étant faits dans un contexte global de productivité, qualité et de coûts (Fig. 2).

L'objectif de cet article est d'une part d'évaluer les possibilités offertes par le tréflage et les trajectoires trochoïdales dans l'usinage d'ébauche d'alliage d'aluminium. Des algorithmes de génération de trajectoires ont été développés et ont pu être optimisés pour présenter le meilleur compromis entre la qualité des surfaces usinées et la productivité associée. Pour cela, les principaux paramètres utiles à la génération de trajectoires de tréflage 

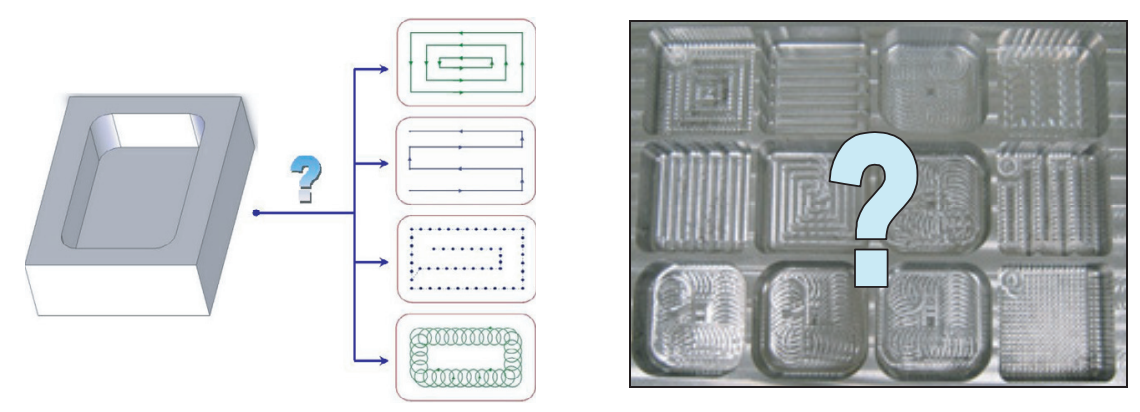

Fig. 2. Choix d'une stratégie pour le vidage de poche.

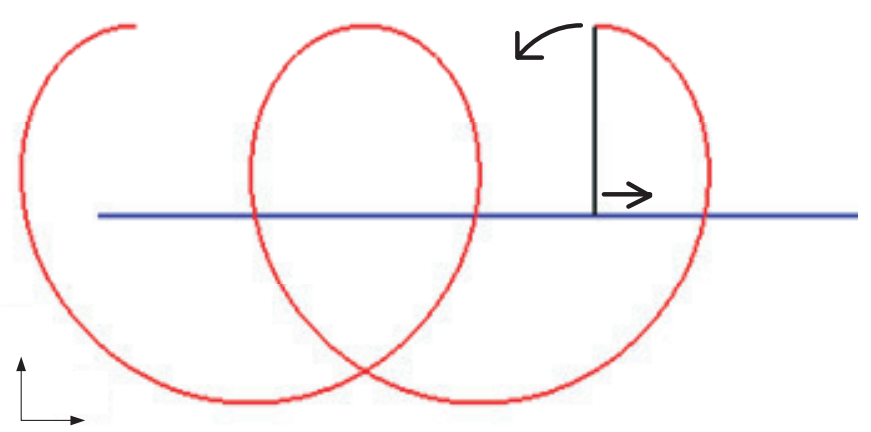

Fig. 3. Génération d'un mouvement trochoïdal.

et d'usinage trochoïdal ont été préalablement déterminés. Ce travail a permis de compenser le manque manifeste des logiciels de génération de trajectoires d'usinage.

D'autre part, une comparaison avec les stratégies classiques a été réalisée. Les trajectoires de tréflage et d'usinage trochoïdal générées ont été testées sur des cas d'usinage réels, le but étant d'établir la fenêtre d'utilisation optimale de chaque stratégie en se basant non seulement sur des critères de temps et de qualité mais aussi sur les performances des moyens disponibles. Les stratégies de tréflage et d'usinage trochoïdal ont ainsi été testées sur deux machines outils : une machine 3 axes sérielle à capacités dynamiques moyennes $\left(0,2 \mathrm{~m} . \mathrm{s}^{-2}\right.$, Num 750$)$ et une machine à structure parallèle 5 axes bénéficiant de capacités dynamiques élevées $\left(50 \mathrm{~m} . \mathrm{min}^{-1}, 15 \mathrm{~m} . \mathrm{s}^{-2}\right.$, Siemens 840D). Les conclusions de cette étude visent à permettre aux programmeurs FAO d'optimiser leurs choix de stratégies de vidage de poche en définissant des conditions de performance pour chacune des stratégies étudiées, en fonction des dimensions de la poche à usiner et des moyens de production disponibles.

De plus, c'est une opportunité de montrer l'intérêt des machines à structure parallèle pour des applications de fraisage. Grâce à leurs capacités dynamiques élevées, elles vont permettre aux industriels d'élargir le spectre de leurs possibilités de production : de nouveaux types de trajectoires vont être capables de rivaliser (voire même de dépasser dans certains cas) les stratégies classiques.

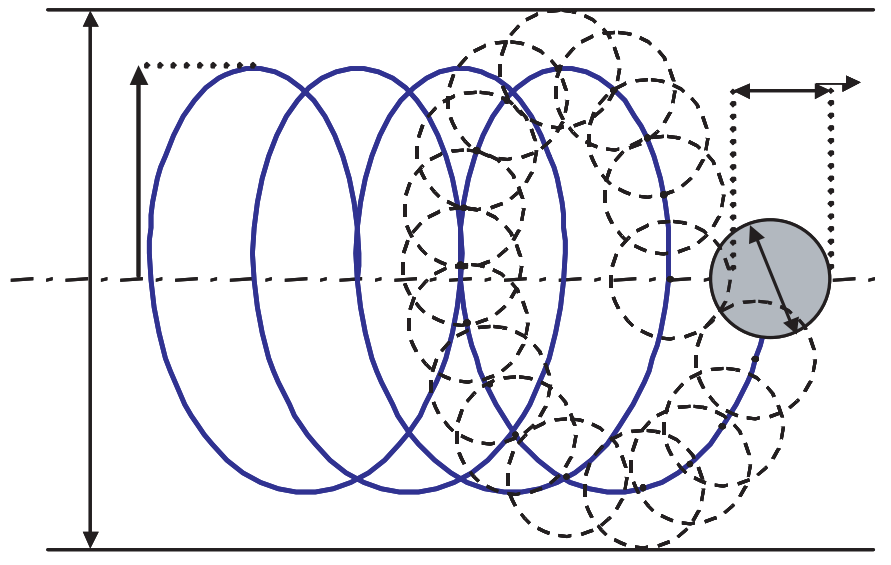

Fig. 4. Paramètres de la trajectoire trochoïdale.

\section{Usinage trochoïdal}

\subsection{Présentation et intérêt}

Une trajectoire trochoïdale est obtenue en combinant un mouvement circulaire uniforme avec un mouvement linéaire uniforme. Par exemple, sur la figure 3, le point B tourne de manière uniforme autour du point $A$, lui-même étant animé d'un mouvement linéaire. Une équation paramétrée d'une trochoïde peut s'écrire de la manière suivante.

$$
\begin{aligned}
& x=V \times t+R \times \cos (\omega . t) \\
& y=R \times \sin (\omega . t)
\end{aligned}
$$

Avec $V$ la vitesse du point A (en m.s $\left.{ }^{-1}\right), R$ la distance entre A et B, $\omega$ la pulsation (par exemple $\omega=$ $2 \pi$ rad. $\left.\mathrm{s}^{-1}\right)$; $t$ représente le temps, il n'a donc pas d'influence sur la forme de la trajectoire.

Pour pouvoir appliquer ce type de trajectoires au fraisage, ces paramètres ont été reliés avec des variables orientées usinage (Fig. 4) : $L$, largeur de la trajectoire enveloppe; $D$, diamètre de l'outil et $\Delta_{\mathrm{t}}$ la prise de passe radiale. Les relations sont les suivantes:

$$
\begin{gathered}
R=\frac{L-D}{2} \\
V=\Delta_{t} \times \frac{\omega}{2 \pi}
\end{gathered}
$$

Une trajectoire trochoïdale peut ainsi être complètement déterminée en utilisant $L, D$ et $\Delta_{\mathrm{t}}$. 
Le principal intérêt des trajectoires trochoïdales est de présenter un rayon de trajectoire constant, l'avance est donc théoriquement uniforme durant l'usinage. De plus, l'épaisseur de copeaux est aussi constante, et le processus d'usinage peut se dérouler dans des conditions favorables (pas de chocs, moins de marquage de la pièce...). Des études de rainurage dans des matériaux durs effectuées en [13] ont même montré une productivité multipliée par 3 par rapport aux stratégies classiques. En effet, en comparaison avec les trajectoires classiques, les forces de coupe et le temps de contact outil-pièce sont moindres. La durée de vie de l'outil peut aussi être augmentée. De la même manière, dans une synthèse sur les avancées en termes de stratégies d'usinage, Marinac voit dans les trajectoires trochoïdales la possibilité d'améliorer la productivité des usinage d'ébauche car elles permettent de maintenir une vitesse d'avance constante durant tout l'usinage [11].

\subsection{Génération de trajectoires}

Les stratégies trochoïdales sont encore peu présentes dans les logiciels de FAO pour la préparation d'opérations de vidage de poches, nous avons donc dû développer nos propres trajectoires. Un algorithme de génération de code ISO 6983 pour le vidage de poches par usinage trochoïdal a donc été créé. Ceci nous a également permis d'optimiser les trajectoires obtenues.

Outre les trois paramètres cités précédemment $(L, D$ et $\Delta_{\mathrm{t}}$ ), les paramètres utiles à la génération de trajectoire sont les dimensions de la poche à vider (longueur, largeur, profondeur), les caractéristiques de l'usinage (profondeur de passe, engagement radial, prise de passe sur un tour lors de la descente). Ainsi, la largeur de la trajectoire enveloppe $L$ est définie comme la moitié de la largeur de la poche pour limiter la longueur du trajet de chaque couche.

Ce qui fait la force de l'usinage trochoïdal est son homogénéité. Il est donc important de ne pas faire apparaître de discontinuité dans le parcours durant les changements de direction voire même pendant les changements de couches d'usinage.

Ainsi la première étape de la construction du parcours consiste à définir la courbe guide donnant la direction générale d'avancement (courbe suivie par le point $\mathrm{A}$ de la Fig. 3). Les équations données en (1) ont donc été modifiées pour permettre plusieurs directions d'avance :

$$
\begin{aligned}
& x(t)=X_{i}+V_{x} \cdot\left(t-t_{i}\right)+R \cdot \cos (\omega \cdot t) \\
& y(t)=Y_{i}+V_{y} \cdot\left(t-t_{i}\right)+R \cdot \sin (\omega \cdot t)
\end{aligned}
$$

L'équation (3) donne cette nouvelle définition pour un tronçon $i$ de la courbe, pour lequel la direction d'avance est la même. L'utilisation d'un décalage d'origine $t_{i}$ permet de raccorder le tronçon à son prédécesseur. La norme du vecteur $V$ donne la prise de passe axiale à chaque boucle. Du fait de la définition de la trochoïde, ces changements de direction ne créeront pas de discontinuité de la courbe. L'usinage d'une couche de la poche sera donc réalisé par une seule et même trochö̈de (Fig. 5).

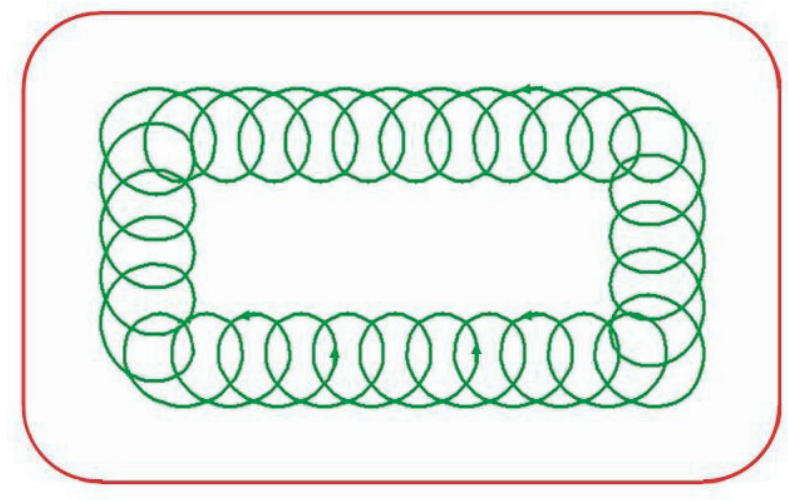

Fig. 5. Exemple de trajectoire d'usinage trochoïdal.

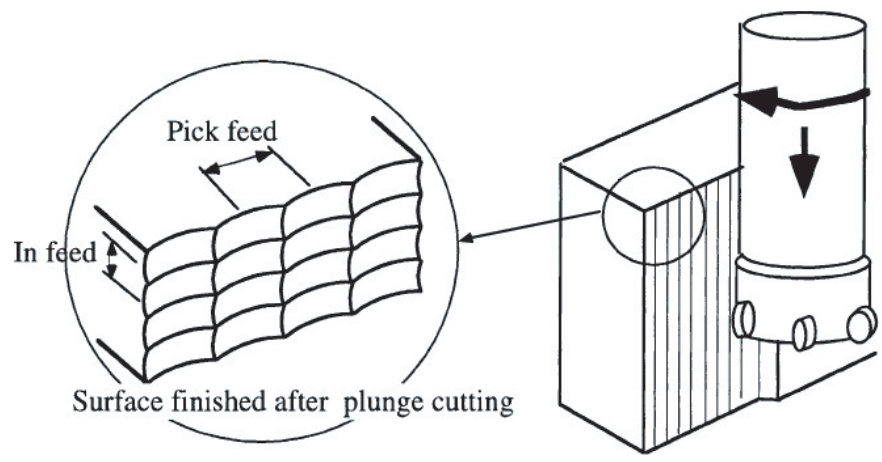

Fig. 6. Principe de l'usinage par tréflage [14].

De plus, la plongée en matière pour atteindre la profondeur de passe souhaitée se fait en utilisant les premières boucles de la trajectoire trochoïdale. À la manière d'une entrée hélicoïdale, un incrément est choisi pour la prise de passe à chaque boucle. En procédant de cette façon, on utilise une unique trochoïde pour vider l'ensemble de la poche.

Une étude préliminaire sur machine outil a conduit à choisir un format de description de type C-splines (permettant de relier des points à l'aide d'une trajectoire de courbure continue) pour définir les parcours : par rapport à l'utilisation de segments de droites (trajectoires en G1), celle-ci nécessite d'une part moins de points pour réaliser la même courbe mais elle génère surtout moins de vibrations.

\section{Tréflage}

\subsection{Présentation et intérêt}

En tréflage, l'outil travaille en effectuant une série de plongées suivant son axe de rotation, la surface usinée est obtenue par superposition de ces «perçages » (Fig. 6).

Les paramètres nécessaires à la définition de trajectoires de tréflage sont (Fig. 7) $\Delta_{\mathrm{p}}$, la prise de passe radiale ; $h$, la hauteur de remontée de l'outil et $d$ la profondeur de la poche. 


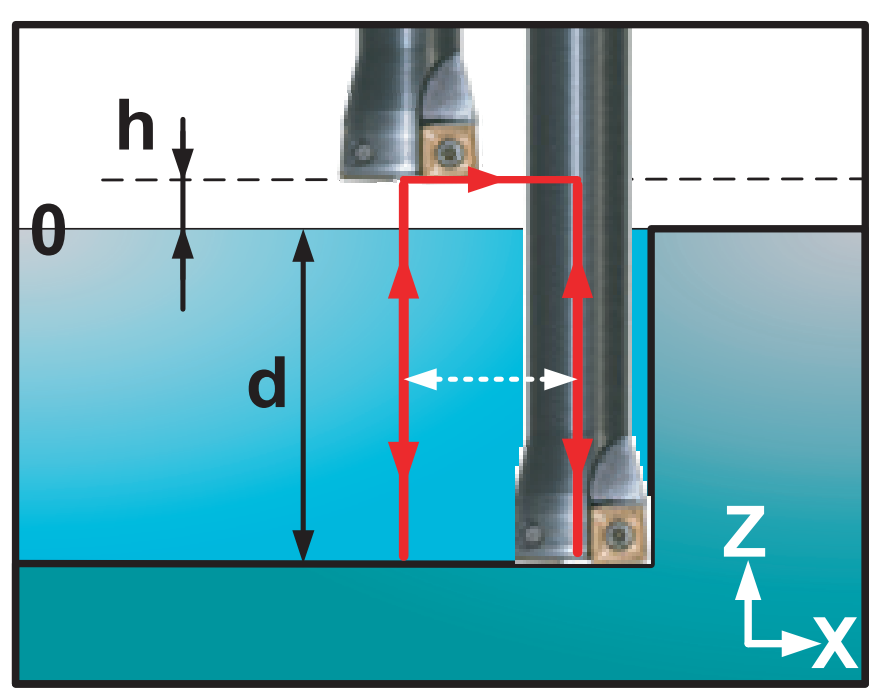

Fig. 7. Paramètres influents en tréflage.

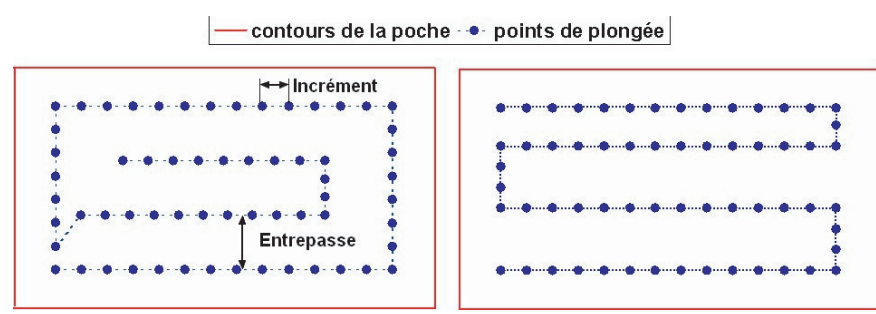

Fig. 8. Exemples de trajectoires de tréflage générées.

D'après [13], le tréflage possède de nombreux avantages étant donné que l'outil travaille dans sa configuration la plus rigide. On limite ainsi la flexion d'outil et le broutement. Dans [14], Wakaoka et al. ont comparé le tréflage à l'usinage en bout lors de l'usinage de parois verticales. Ils ont conclu que le tréflage apporte un meilleur positionnement et une meilleure rugosité de la surface par rapport à l'usinage en bout. Une comparaison du tréflage et de l'usinage en bout est également proposée par Al-Ahmad dans [15]. Elle se limite uniquement à des aspects théoriques sans validation expérimentale mais identifie certains apports possibles du tréflage.

L'apport des trajectoires de tréflage pour l'usinage d'ébauche a également été présenté par Zelinski dans [12]. En s'appuyant sur un retour d'expérience industriel, il indique que le temps plus long passé à programmer les trajectoires est largement compensé par les gains obtenus pour les temps de fabrication, si bien que le tréflage est devenu indispensable à l'augmentation de la productivité.

\subsection{Génération de trajectoires}

Comme les trochoïdes, les trajectoires de tréflage ne sont que peu voire pas proposées par les logiciels de FAO. Un algorithme spécifique a donc été développé. En plus des paramètres cités précédemment, celui-ci demande à l'utilisateur de choisir l'incrément maximal autorisé entre deux plongées ainsi que l'entrepasse maximale (Fig. 8). L'incrément maximal sert à définir le nombre de plongées
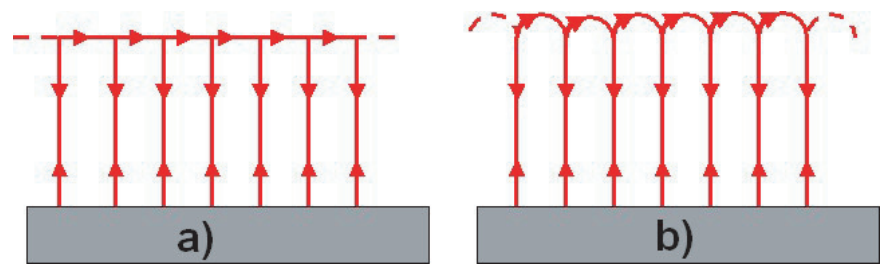

Fig. 9. Optimisation de la trajectoire de tréflage.

de chaque segment du parcours dans le plan $X Y$. En pratique, l'incrément utilisé répartit ces points de manière uniforme sur le segment. Il sera donc inférieur à la valeur maximale et permet d'optimiser la matière enlevée à chaque plongée.

Deux types de trajectoires sont aussi proposés dans le but d'optimiser le couple productivité-qualité. Le premier type s'appuie sur un parcours en colimaçon pour les déplacements dans le plan $X Y$. En réglant l'incrément maximal, l'utilisateur peut ainsi choisir la hauteur de crête des parois de la poche. Le second type s'appuie plutôt sur un parcours zigzag dans le plan $X Y$. On privilégie ici la productivité aux dépens d'une hauteur de crête irrégulière sur les parois (Fig. 8).

Un autre aspect développé concerne les déplacements hors matière. Ainsi, une étude expérimentale sur machine a montré que le programme d'usinage s'exécute plus vite si le raccordement entre deux plongées consécutives s'effectue à l'aide de demi-cercles. En effet, il n'y a alors pas de discontinuité de vitesse ni d'accélération au niveau des axes de la machine. La trajectoire b) de la figure 9 est donc plus rapide que la trajectoire a), bien que la distance à parcourir soit plus longue. C'est elle qui sera donc utilisée dans l'algorithme de génération de parcours.

\section{Comparaison des stratégies lors du vidage d'une poche}

Une campagne de tests visant à comparer les trajectoires de tréflage et d'usinage trochoïdal développées avec les trajectoires classiques utilisées pour le vidage de poche a été menée. Les résultats d'une étude préliminaire ont montré que les parcours zigzag sont plus productifs pour l'usinage d'ébauche que les parcours en contours parallèles tout en conservant une qualité équivalente. L'étude expérimentale s'est donc focalisée sur le tréflage, l'usinage trochoïdal et sur le zigzag.

Chaque stratégie a été testée sur une poche de dimensions $160 \times 100 \times 40(L \times l \times h)$ usinée dans un alliage d'aluminium de type aéronautique. La plupart de l'étude a été réalisée sur une machine outil 5 axes à structure parallèle disposant de bonnes capacités dynamiques (50 m.min ${ }^{-1}, 15$ m.s ${ }^{-2}$, Siemens 840D). Ensuite, certaines poches ont été usinées sur une machine 3 axes sérielle avec des capacités plus modestes $\left(0,2\right.$ m.s ${ }^{-2}$, Num 750), ceci afin d'évaluer l'influence de la dynamique de la machine sur l'efficacité des stratégies employées. Deux outils de diamètre $32 \mathrm{~mm}$ et à 2 dents ont été utilisés, le premier pour le tréflage, l'autre pour les autres trajectoires. 
Tableau 1. Paramètres de coupe des stratégies testées.

\begin{tabular}{|c|c|c|c|c|}
\cline { 2 - 5 } \multicolumn{1}{c|}{} & $\begin{array}{c}\text { Vitesse de coupe } \\
\mathrm{m}_{\mathrm{cmin}} \text { m }^{-1}\end{array}$ & $\begin{array}{c}\text { Avance } \\
\text { mm.dent }^{-1}\end{array}$ & $\begin{array}{c}\text { Incrément axial } \\
\mathrm{mm}\end{array}$ & $\begin{array}{c}\text { Profondeur de passe } \\
\mathrm{mm}\end{array}$ \\
\hline tréflage & 2413 & 0,35 & 11 & 40,00 (prof. de poche) \\
trochoides-1 & 2413 & 0,35 & 8 & 6,66 \\
trochoides-2 & 2413 & 0,35 & 2 & 13,50 \\
zigzag-1 & 2413 & 0,35 & 25 & 6,66 \\
zigzag-2 & 1508 & 0,26 & 25 & 6,66 \\
\hline
\end{tabular}

\section{Débit de copeaux (L/min)}

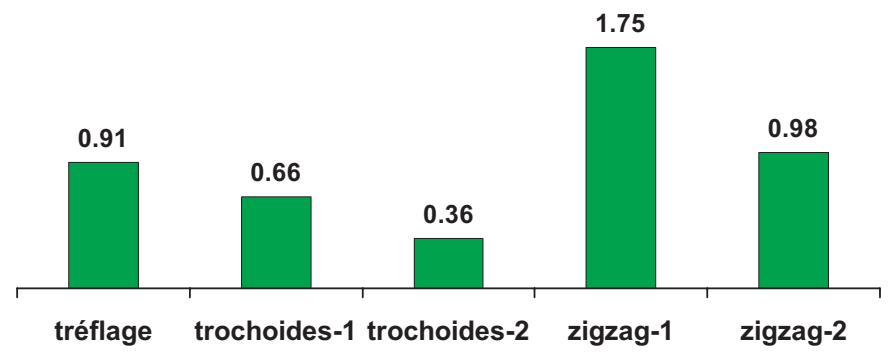

Fig. 10. Débit de copeaux associé à chaque stratégie.

Les conditions de coupe ont été choisies de manière à se trouver au maximum des capacités des outils fournies par le carburier, bien que ces valeurs soient plutôt adaptées aux stratégies classiques. La vitesse de coupe ainsi que l'avance ont été les mêmes pour toutes les poches sauf pour celle appelée zigzag- 2 , où les valeurs employées ont été celles préconisées par le fournisseur pour des performances idéales. Le tableau 1 présente les conditions de coupe utilisées pour chaque stratégie.

\subsection{Analyse de la productivité}

Les conditions d'usinage ont été les mêmes pour toutes les poches. La figure 10 donne le débit de copeaux associé à chaque stratégie.

On constate que la stratégie zigzag fournit la meilleure productivité, du fait que les parcours utilisés sont les plus courts. L'outil effectue un trajet de $4400 \mathrm{~mm}$ pour vider toute la poche. Cette valeur est de $5680 \mathrm{~mm}$ pour le tréflage et de $15270 \mathrm{~mm}$ pour les trochoïdes. Cette différence s'explique par le fait que le parcours en zigzag limite fortement les déplacements hors matière : l'outil travaille sur $80 \%$ du trajet. Pour l'usinage trochoïdal l'outil travaille uniquement sur $55 \%$ du trajet. Cette valeur tombe même à $25 \%$ pour le tréflage.

\subsection{Analyse des forces de coupe}

En tréflage, le relevé de force sur la poche complète montre une excellente répétabilité. On ne s'intéressera donc qu'aux résultats d'un seul cycle (une plongée selon $Z$, une remontée, un déplacement jusqu'au point de plongée suivant).

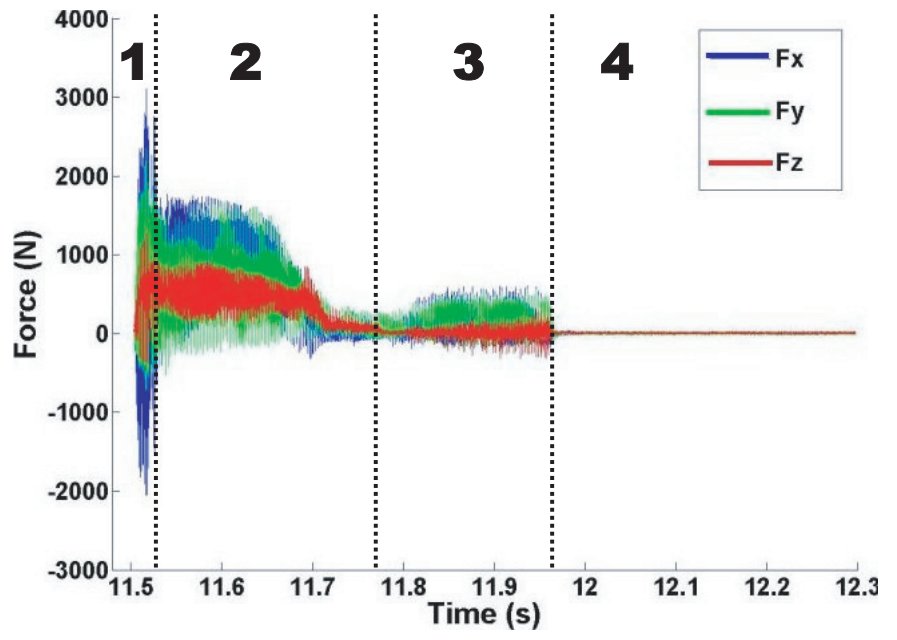

Fig. 11. Mesures de force pour un cycle de tréflage.

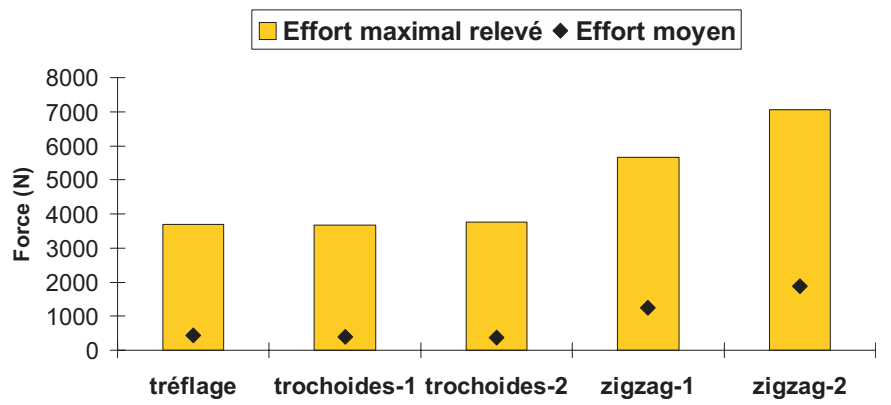

Fig. 12. Comparaison des efforts nécessaires pour chaque stratégie.

Au niveau des efforts, un cycle de tréflage peut être divisé en 4 phases (Fig. 11) :

1. entrée en matière : à cause des vibrations ou d'une trop faible hauteur de coupe, les forces relevées sont supérieures à celles des autres étapes;

2. descente selon $Z$ : l'outil travaille avec les conditions de coupe programmées;

3. remontée selon $Z$ : l'outil frotte sur la surface usinée lors de phase 2 ;

4. déplacement hors matière pour atteindre le point de plongée suivant.

Le principal résultat qui apparaît est que, contrairement aux idées reçues, les efforts selon l'axe $Z$ sont inférieurs à ceux dans le plan $X Y$, pour les conditions de coupe choisies. De plus, si l'on souhaite un meilleur état 


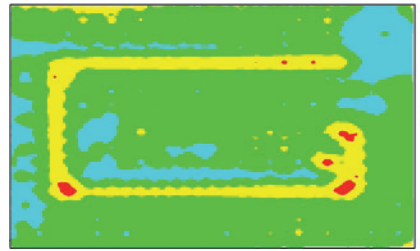

Tréflage

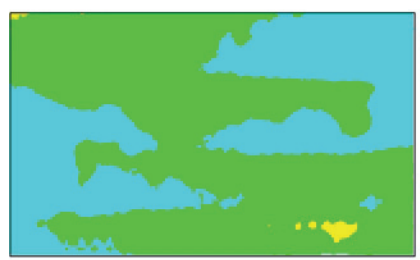

Zigzag-1

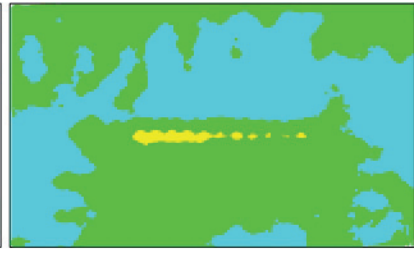

Trochoïdes-1

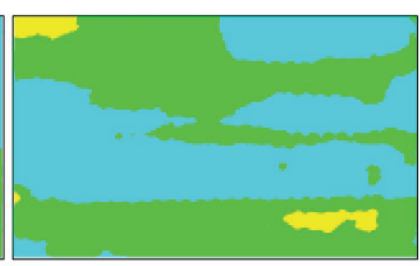

Zigzag-2

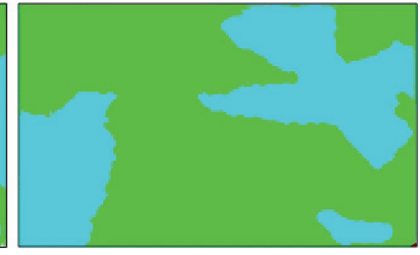

Trochoïdes-2

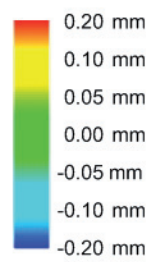

Fig. 13. Planéité en fond de poche.

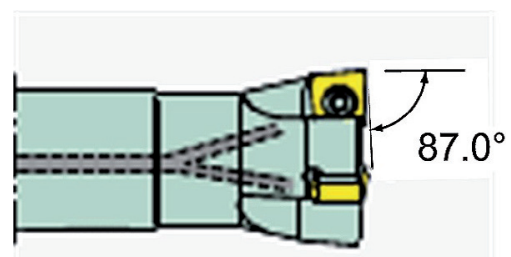

Fig. 14. Détail de l'outil de tréflage.

de surface, la phase 3 peut être supprimée en déplaçant légèrement l'axe de l'outil lors de la remontée.

En usinage trochoïdal, l'outil ne travaille que $55 \%$ du temps et on observe une grande différence entre l'effort maximal relevé $(3886 \mathrm{~N})$ et l'effort moyen durant l'usinage (386 N). Ceci explique que ce type de parcours sollicite moins la pièce et l'outil.

Les relevés d'effort en zigzag montrent que l'outil est globalement plus sollicité en utilisant ces stratégies.

Un comparatif des résultats d'efforts obtenus est présenté sur la figure 12. Bien que les paramètres de coupe aient été les mêmes pour tous les parcours, le tréflage et l'usinage trochoïdal sont beaucoup moins gourmands en effort que le zigzag, en particulier pour les efforts moyens.

\subsection{Qualité de la poche usinée}

Le fond de chaque poche a été inspecté sur Machine à Mesurer Tridimensionnelle pour quantifier le défaut de planéité (Fig. 13).

L'erreur la plus importante $(0,3 \mathrm{~mm})$ est obtenue pour le tréflage, mais cela vient essentiellement de la géométrie de l'outil utilisé : comme indiqué sur la figure 14, l'angle entre le bas de la plaquette et l'axe de l'outil est à $87^{\circ}$, de manière à optimiser le travail de l'outil : la surface générée par une plongée d'outil en fond de poche n'est pas plane.

Les trochoïdes présentent la meilleure planéité de fond de poche. Elle est la meilleure pour la poche usinée avec un faible incrément axial : l'outil balaie plusieurs fois la même zone, ce qui supprime les crêtes liées à l'usinage.

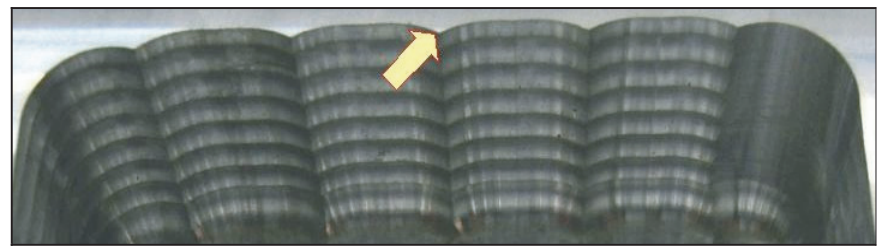

Fig. 15. Exemple de crêtes sur les parois en zigzag.

Les poches usinées en zigzag disposent également d'une qualité suffisante, l'erreur de planéité étant inférieure à $0,1 \mathrm{~mm}$. Tout comme pour les trochoïdes, on pourra s'affranchir d'une finition du fond de poche.

Concernant les parois verticales, toutes les stratégies offrent de bons résultats de perpendicularité et d'état de surface. Le zigzag présente malgré tout l'inconvénient de laisser une crête importante $(6 \mathrm{~mm})$ entre deux passes d'usinage, une finition est donc nécessaire (Fig. 15). Le tréflage est le meilleur choix vis-à-vis de ce critère. De plus, l'algorithme de génération utilisé permet de choisir l'incrément radial pour contrôler la hauteur de crête sur les parois.

\subsection{Influence et importance de la dynamique de la machine}

Une comparaison du temps théorique, où la trajectoire est entièrement parcourue à la vitesse programmée, et du temps réel, mesuré lors de l'usinage sur la machine à structure parallèle a été effectuée. C'est dans le cas du tréflage que l'écart est le plus important avec une marge d'erreur de $50 \%$. Cela vient du fait que cette stratégie présente beaucoup de discontinuités, en particulier sur l'axe $Z$, où on observe une inversion du vecteur vitesse lorsque l'outil amorce sa remontée. Il est donc important de disposer d'une dynamique élevée (au sens vitesse, accélération et jerk) en ayant une commande numérique adaptée à ces performances pour que cette stratégie soit efficace. Pour le zigzag, la marge d'erreur n'est que de $33 \%$ vu que les discontinuités n'apparaissent qu'au niveau des parois 


\section{Ecart tps_théorique / tps_réel}

$\square$ PKM 5 axes $\square$ SKM 3 axes

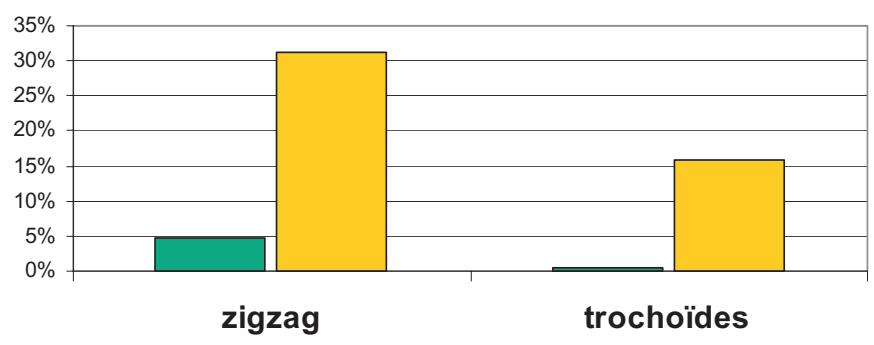

Fig. 16. Comparaison de deux machines outils.

de la poche. Mais c'est en utilisant des trochö̈des que cette erreur est la plus faible : temps théorique et réel sont comparables à moins de $1 \%$. On peut expliquer cela par le fait que le mouvement trochoïdal se rapproche du mouvement circulaire uniforme à accélération centripète et valeur constante.

Les trajectoires de zigzag et de trochoïdes ont ensuite été testées sur la machine sérielle 3 axes. Pour un même programme d'usinage, la valeur d'erreur de $1 \%$ en trochoïdes passe à plus de $15 \%$. Les résultats sont similaires pour les parcours zigzag (Fig. 16). L'influence de la dynamique de la machine est donc très importante sur la productivité d'une stratégie d'usinage.

Le tréflage a également été testé sur la machine sérielle, mais l'axe $Z$, pas assez dynamique, n'a pas pu suivre les ordres du DCN et l'outil n'est pas allé assez profondément dans la matière. La poche n'a pas pu être usinée.

Une dernière expérience a montré l'influence du jerk. Une poche a été usinée sur la machine parallèle pour un parcours trochoïdal avec un jerk réduit à $5 \mathrm{~m} . \mathrm{s}^{-3}$ (contre $100 \mathrm{~m} . \mathrm{s}^{-3}$ habituellement). L'écart entre temps théorique prévu (FAO) et temps réel est passé au-dessus des $35 \%$.

\subsection{Synthèse des résultats obtenus}

Les résultats expérimentaux montrent le réel potentiel des stratégies de tréflage et d'usinage trochoïdal face aux parcours classiques. La première chose à remarquer est l'importance de la dynamique de la machine. Ainsi, les nouvelles stratégies n'ont aucun avantage, dans les cas traités, quand les capacités d'accélération des axes sont en dessous de $10 \mathrm{~m} . \mathrm{s}^{-2}$.

En regardant les pièces tests, on remarque aussi aisément que l'usinage trochoïdal ne peut pas défier le tréflage ni le zigzag en termes de productivité. Le parcours est beaucoup trop long. Néanmoins, si la qualité de la poche à produire est le critère prépondérant, les trochoïdes sont recommandées. On peut même contrôler les efforts de coupe en agissant sur l'incrément radial. Ces stratégies sont sûrement plus profitables pour des matériaux durs.

La figure 17 montre le débit de copeaux associé à chaque stratégie dans le but d'obtenir une poche de même

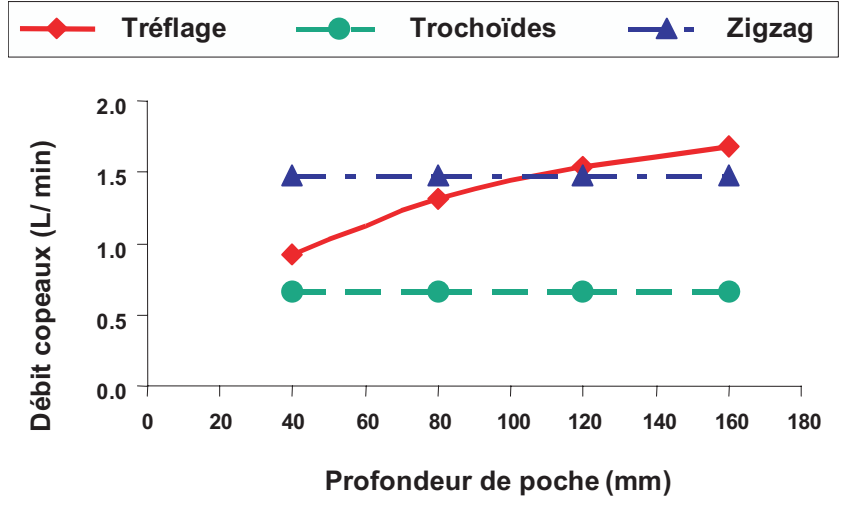

Fig. 17. Évolution du débit de copeaux pour une poche de $160 \times 100 \mathrm{~mm}$.

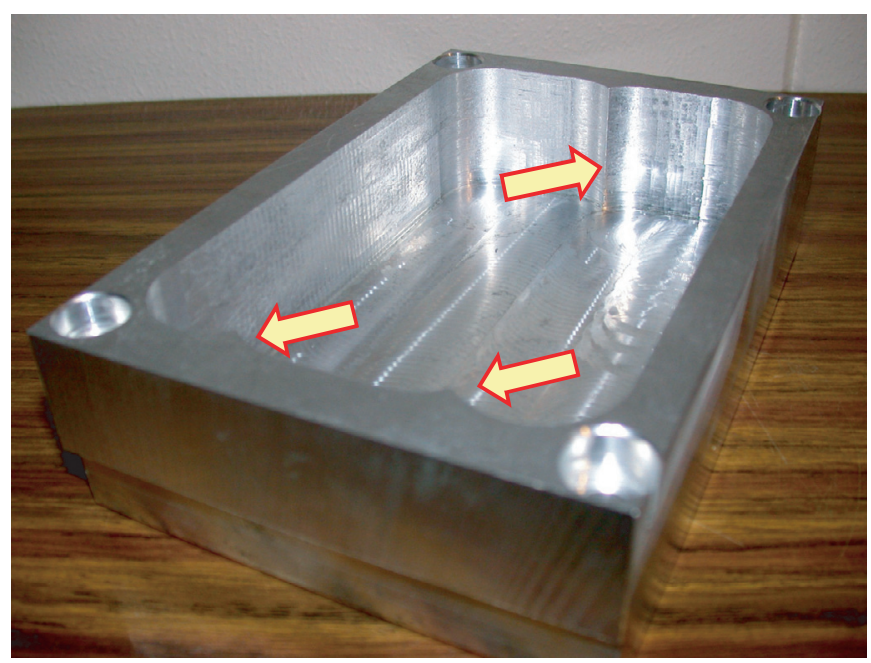

Fig. 18. Poche usinée avec un trajet en zigzag.

qualité (par exemple, le parcours en zigzag est complété par un contournage pour écrêter les parois). On remarque ainsi que, contrairement aux autres stratégies, la productivité du tréflage dépend de la profondeur de la poche à vider. Elle devient la meilleure stratégie dès que la profondeur de la poche dépasse sa largeur. De plus, le débit de copeaux n'est pas affecté par la présence d'un îlot lorsqu'on utilise le tréflage. On pourra même s'affranchir d'une finition en utilisant un outil à fond plat et en choisissant convenablement l'incrément radial.

Les stratégies en zigzag, quant à elles, génèrent les parcours les plus courts, ce qui leur assure une très bonne productivité. Leurs deux principaux inconvénients sont la nécessité d'utiliser un contournage pour éliminer les crêtes restantes sur les parois (Fig. 18) et la relative importance des efforts de coupe, comparativement aux autres stratégies. Elles sont donc adaptées à des poches peu profondes.

\section{Conclusion}

Dans cet article, les forces et faiblesses des stratégies de tréflage et d'usinage trochoïdal ont été étudiées. Une 
première étude a permis de développer des algorithmes de génération de trajectoires optimisées destinées au vidage de poches. Une étude expérimentale permet de comparer ces stratégies aux parcours « classiques ». Des domaines d'emploi privilégiés pour chaque type de trajectoires ont été déterminés. L'importance de la dynamique de la machine outil a également été soulignée.

Le vidage de poches ne possède pas de solution optimale unique, mais son optimisation sera fonction de la morphologie de la pièce, des moyens disponibles et de l'objectif à atteindre.

Remerciements. Ce travail a été conduit dans le cadre du groupe de travail Manufacturing 21 qui regroupe 12 laboratoires de recherche français. Les thèmes étudiés sont la modélisation du processus de fabrication, l'usinage virtuel et le développement de nouvelles méthodes de fabrication.

\section{Références}

[1] C.A.H. Lambregts, F.L.M. Delbressine, W.A.H. de Vries, A.C.H. van der Wolf, An efficient automatic tool path generator for D free-form pockets, Computers in Industry 29 (1996) 151-157

[2] M. Held, Voronoi diagrams and offset curves of curvilinear polygons, Computer-Aided Design 30 (1998) 287300

[3] B.K. Choi, B.H. Kim, Die-cavity pocketing via cutting simulation, CAD Computer Aided Design 29 (1997) 837846

[4] B.K. Choi, S.C. Park, Pair-wise offset algorithm for 2D point-sequence curve, CAD Computer Aided Design 31 (1999) 735-745
[5] V. Pateloup, E. Duc, P. Ray, Corner optimization for pocket machining, Int. J. Machine Tools and Manufacture 44 (2004) 1343-1353

[6] K. Tang, S.-Y. Chou, L.-L. Chen, An algorithm for reducing tool retractions in zigzag pocket machining, Computer-Aided Design 30 (1998) 123-129

[7] M. Bieterman, T.B. Company, Industrial Problems Seminar, Mathematics \& Computing Technology, University of Minnesota IMA, 2001, CD-rom

[8] K. Mawussi, S. Lavernhe, C. Lartigue, Usinage de poches en UGV, Aide au choix de stratégies, Revue de CFAO et d'informatique graphique 18 (2004) 337-349

[9] B.H. Kim, B.K. Choi, Machining efficiency comparison direction-parallel tool path with contour-parallel tool path, Computer-Aided Design 34 (2002) 89-95

[10] M. Terrier, A. Dugas, J.-Y. Hascoet, Qualification of parallel kinematics machines in high-speed milling on free form surfaces, Int. J. Machine Tools Manufacture 44 (2004) 865-877

[11] D. Marinac, Tool path strategies for high speed milling, MMS Online, http://www.mmsonline.com/articles/ 020004.html, 2001

[12] P. Zelinski, Plunge Roughing Right Now, MMS Online, http://www.mmsonline.com/articles/100001.html, 2001

[13] S. Guerin, Nouvelles stratégies d'usinage en ébauche, trochoïdes et tréflage, CETIM, 2004

[14] S. Wakaoka, Y. Yamane, K. Sekiya, N. Narutaki, Highspeed and high-accuracy plunge cutting for vertical walls, J. Mat. Proc. Tech. 127 (2002) 246-250

[15] M. Al-Ahmad, A. D'Acunto, C. Lescalier, O. Bomont, Modeling of cutting forces in plunge milling, Advanced Manufacturing Systems and Technology 486 (2005) 155164 